\title{
Human-enabled Edge Computing: Exploiting the Crowd as a Dynamic Extension of Mobile Edge Computing
}

\author{
Paolo Bellavista ${ }^{1}$, Stefano Chessa ${ }^{2,3}$, Luca Foschini ${ }^{1}$, Leo Gioia ${ }^{1}$, Michele Girolami ${ }^{3}$ \\ ${ }^{1}$ Dipartimento di Informatica: Scienza e Ingegneria (DISI), University of Bologna \\ Viale Risorgimento, 2, 40136 Bologna, ITALY - Ph.: +39-051-20-93001 \\ ${ }^{2}$ Dipartimento di Informatica, University of Pisa \\ Largo B. Pontecorvo, 356127 Pisa \\ ${ }^{3}$ National Council of Research, ISTI-CNR,Via G. Moruzzi 156124 Pisa \\ email: stefano.chessa@unipi.it, \{paolo.bellavista, luca.foschini\}@unibo.it, \\ leo.gioia@studio.unibo.it, michele.girolami@isti.cnr.it
}

\begin{abstract}
The Mobile Edge Computing (MEC) vision leverages the availability of powerful and low-cost middle boxes, statically deployed at suitable edges of the network and acting as local proxies for the centralized cloud backbone; this potentially enables, among the others, better scalability and better reactivity in the interaction with mobile nodes via local control decisions and actuation. MEC has already been proposed as an enabler for several Internet-of-Things and Cyber-Physical Systems application scenarios, but mutual benefits due to the integration of MEC and Mobile CrowdSensing (MCS). The paper originally proposes Human-driven Edge Computing (HEC) as a new model to ease the provisioning and to extend the coverage of traditional MEC solutions. From a methodological perspective, we show how it is possible to exploit MCS i) to support the effective deployment of Fixed MEC (FMEC) proxies and ii) to further extend their coverage through the introduction of impromptu and human-enabled Mobile MEC $\left(\mathrm{M}^{2} \mathrm{EC}\right)$ proxies. In addition, we describe how we have implemented these novel concepts in the MCS ParticipAct platform through the integration of the MEC Elijah platform in the ParticipAct living lab, an ongoing MCS real-world experiment that involved about 170 students of the University of Bologna for more than two years. Reported experimental results quantitatively show the effectiveness of the proposed techniques in elastically scaling the load at edge nodes according to runtime provisioning needs.
\end{abstract}

\section{Introduction}

Mobile Edge Computing (MEC) is a recent architectural model and specification proposal (i.e., by European Telecommunications Standards Institute - ETSI) that aims at evolving the traditional two-layers cloud-device integration model, where mobile nodes directly communicate with a central 
cloud through the Internet, with the introduction of a third intermediate middleware layer that executes at so-called network edges. This promotes a new three-layer device-edge-cloud hierarchical architecture, which is recognized as very promising for several application domains [1]. In fact, the new MEC model allows moving and hosting computing/storage resources at network edges close to the targeted mobile devices [1].

In MEC solutions, edges are commonly deployed statically (by telco providers) and logically coupled with Radio Access Network (RAN) base stations (i.e., being deployed close to either the eNodeB or the Serving Gateway); we claim that such model still faces with some weaknesses. First, the number of edges is generally limited, since edges introduce additional costs of operation for the supported services, such as deployment and maintenance. Second, edges are rarely are re-deployed and this might be highly inefficient. Finally, some geographical areas might be highly populated and interesting for a service only during specific time slots (daily, weekly, or yearly patterns).

At the same time, the worldwide of smartphones has recently boosted Mobile CrowdSensing (MCS) as the possibility to leverage people roaming though the city with their sensor-rich devices as intelligent sensing probes. In fact, by installing an MCS application, any smartphone can become part of a large-scale sensor network. However, for some high-demanding MCS services (e.g., a surveillance application monitoring an environment with smartphone cameras and implementing face recognition to trace suspicious users' movements), regular smartphones often have not enough capabilities to timely perform the requested local tasks (e.g in hostile environments with disconnections from the cloud).

In other words, we claim that there are several practical cases of large and growing relevance where the joint exploitation of MEC and MCS solutions would bring highly significant benefits in terms of efficient resource usage and perceived service quality. To the best of our knowledge, only a very limited number of seminal works has explored the mutual advantages in the joint use of these two classes of solutions, and they are mostly focused on pure technical communication aspects without considering the crucial importance of having humans as central contributors in the loop [2, $3,4]$.

The paper aims to fill that gap and proposes Human-driven Edge Computing (HEC) as a new model to ease the provisioning and deployment of MEC platforms as well as to enable more powerful MEC-enabled MCS applications by contributing with several core elements of technical novelty. First and foremost, HEC eases the planning and deployment of the basic MEC model: it mitigates the potential weaknesses of having only Fixed MEC entities (FMEC) by exploiting MCS to continuously monitor humans and their mobility patterns, as well as to dynamically re-identify hot locations of potential interest for the deployment of new edges. Second, to overcome FMEC 
limitations, HEC enables the implementation and dynamic activation of impromptu and temporary Mobile MEC entities $\left(M^{2} E C\right)$ that leverage resources of locally available mobile devices. Hence, a $\mathrm{M}^{2} \mathrm{EC}$ is a local middleware proxy dynamically activated in a logical bounded location where people tend to stay for a while in a repetitive and predictive way [5], thus realizing a mobile, opportunistic, and participatory edge node. Third, given that $\mathrm{M}^{2} \mathrm{EC}$, differently from FMEC, do not implement powerful backhaul links toward the core cloud, HEC exploits local one-hop communications and the store-and-forward principle by using humans (moving with their devices) as VM/container couriers to enable migrations between well-connected FMEC and local $\mathrm{M}^{2} \mathrm{EC}$.

Finally, the paper also originally contributes to the literature in the field by presenting the new HEC model implemented into the real-world ParticipAct MCS living lab [6], i.e., an experiment that involved about 170 students of the University of Bologna for more than two years. This large scale experiment allowed us to practically characterize $\mathrm{M}^{2} \mathrm{EC}$ locations and to collect interesting performance indicators about the improvements obtainable via HEC, as well as its drawbacks in terms of stability of $\mathrm{M}^{2} \mathrm{EC}$, opportunistically enabled thanks to the participation and contribution of humans-in-the-loop, and possible connectivity issues between FMEC and $\mathrm{M}^{2} \mathrm{EC}$.

\section{Background and Related Work}

Without claiming completeness, this section first reports some needed background about both MEC and the usage of human mobility features to efficiently enable the new HEC scenario, then it briefly overviews the current state-of-the-art in these very active fields.

\subsection{Mobile Edge Computing Architecture and its Human-enabled Extension}

Our reference scenario is shown in Fig. 1 and enriches the usual three-layer device-MEC-cloud hierarchical architecture (based on the interposition of FMEC entities) by adding new impromptu and temporarily active $\mathrm{M}^{2} \mathrm{EC}$ entities. Indeed, the use of MCS and the continuous tracing of humans volunteering to the completion of MCS tasks (monitoring both their mobility and their performance in terms of completion rates of assigned sensing tasks) enable: i) the identification of the best locations where users tend to aggregate more, thus easing the effective deployment of FMEC and $\mathrm{M}^{2} \mathrm{EC}$; ii) the selection of the users who should be more willing to host $\mathrm{M}^{2} \mathrm{EC}$ acting as local access points to the hierarchical HEC.

To make a simple example, we learned from ParticipAct MCS living lab that there are some aggregation zones through most of the day (they are good candidates as FMEC, see $E_{1}, E_{2}$, and $E_{3}$ in Fig. 1), while there are other areas that are populated only during shorter and different timeslots (e.g., $\mathrm{P}_{1}$ and $\mathrm{P}_{4}$ from 9:00AM to 10:30AM, while $\mathrm{P}_{2}$ is frequented only from 4:00PM to 6:00PM). These latest areas, out of the highly frequented people paths, would highly benefit of being served 
by a local (in time and space) $\mathrm{M}^{2} \mathrm{EC}$, while it would be inefficient and overprovisioned to have additional FMEC there (see Fig. 1).

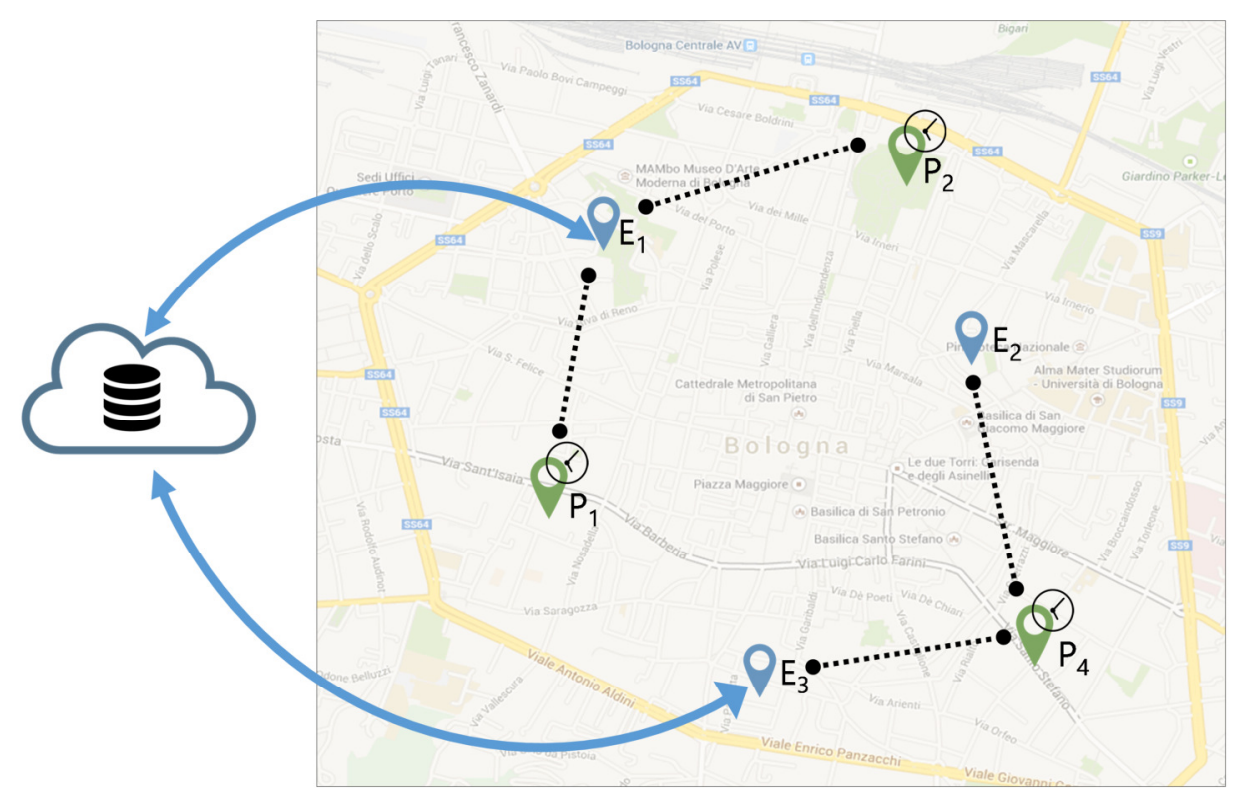

Figure 1. FMEC, $\mathrm{M}^{2} \mathrm{EC}$, and couriers in our HEC model.

Moreover, from the Mobile Social Networks (MSN) research we borrow the key idea of exploiting opportunistic interactions among devices to share Virtual Machines (VM)/containers that have to be migrated by couriers from/to FMEC (see Fig. 1) [7]. We assume one-hop direct mode communications that can span from short Bluetooth coverage (i.e., up to $25 \mathrm{~m}$ ), to broader WiFidirect (i.e., up to $150 \mathrm{~m}$ ) and LTE-direct (i.e., up to 500m) ones. Similarly to MSN, we consider that courier devices automatically down/upload VM/containers from the FMEC as soon as they are close to them; in turn, they share the data gathered with devices roaming in the same $\mathrm{M}^{2} \mathrm{EC}$ (see dotted lines in Fig. 1).

Let us note that the goal of this paper is assessing the feasibility of the solution proposed. Accordingly, in our experimental results, adopting an assumption typical of the MSN research area [7], we will consider an ideal situation where nodes within transmission ranges can communicate while, also due to space limitations, we leave a thorough analysis of the issues related to wireless medium interferences, network congestion, and bandwidth allocation for moving data from/to the FMECs and $\mathrm{M}^{2} \mathrm{ECs}$ as part of our future work. As for the description of the algorithms used to identify FMEC and $\mathrm{M}^{2} \mathrm{EC}$, as well as most suitable human couriers, we refer to the next section. 


\subsection{Related Work}

One of the key open challenges in MCS is the possibility of exploiting socio-technical network effects to consolidate and extend the crowd by leveraging communities of volunteers [6]. In the present work, we leverage these new research trends at the crossroads of MCS and MSN to facilitate the identification and formation of $\mathrm{M}^{2} \mathrm{EC}$. In addition to the strong novelty in terms of HEC model, what makes our research efforts unique is also the fact that the ParticipAct living lab [6] mimics closely a very realistic deployment MEC scenario [6].

Focusing on architectural aspects of HEC, the MEC/fog literature has already produced some relevant modeling work and some seminal design/implementation results. Narrowing to efforts close to ours, as reported in [1], some first exploratory research activities have considered cooperation issues between edges and the core, but only a very few works concentrated on the opportunities of having cooperation between devices and the edges. Considering MCS as application scenario, [2] and [3] propose to enhance the MCS process by leveraging intermediate MEC nodes, namely, FMECs, to boost data upload from mobile nodes to the infrastructure [2] and to provide more computing/storage capabilities closer to end mobile devices [3]. To address relevant energy efficiency issues, [8] proposes an interesting approach based on game-theory in order to select task and manage the scheduling of the edge of the network by introducing the notion of Container-as-aService. A very recent and interesting work, the closest to our HEC concept for what relates to enabling more collaboration between entities co-located at edges is [4]: it proposes not only to have the traditional "vertical" collaboration between devices, MEC, and cloud level, but also an "horizontal" collaboration between entities at the same level via ad-hoc communications; however, it neglects humans and social/mobility effects, namely, there is no idea to dynamically identify and impromptu form $\mathrm{M}^{2} \mathrm{ECs}$ as in our novel HEC proposal.

Concerning the system and implementation aspects only a very few research activities have focused on VM/container migration on MEC middleware for mobile services over hostile environments, which is a relevant challenging aspect of modern CPS. [9] highlights the limitations of traditional live VM migration on edge devices, by proposing live migration in response to client handoff in cloudlets, with less involvement of the hypervisor and by promoting migration to optimal offload sites, adapting to changing network conditions and processing capacity. [10] presents the foglets programming infrastructure that handles mechanisms for quality/workload-sensitive migration of service components among fog nodes. [11] proposes the usage of cloudlets to support mobile multimedia services and adjusts resource allocation triggered by runtime handoffs. [12] evaluates handoff conditions in relation to various parameters (e.g., signal strength, bit rate, number of interactions between cloudlets and associated devices) and decides to offload the computation 
based on fuzzy techniques. [13] proposes a multi-agent-based code offloading mechanism, by using reinforcement learning and code blocks migration, to reduce both execution time and energy consumption of mobile devices. While these papers explore the highly relevant issue of integrated management of handover operations with $\mathrm{VM}$ /container migration, none of them explore the possibility to use humans as storage/VM/container carriers.

\section{Extending Mobile Edge Computing through the Crowd}

This section first overviews the HEC distributed architecture and its main components and functions, and then it presents the main guidelines and engineering tradeoffs we implement for the selection of FMEC, $\mathrm{M}^{2} \mathrm{EC}$, and human couriers.

\subsection{Architecture and some Implementation Insights about our Original HEC Middleware}

HEC extends the emerging MEC three-layer hierarchical architecture through the distinction of two types of MECs, namely FMEC and $\mathrm{M}^{2} \mathrm{EC}$. In the following, we present the main functions of our proposed HEC middleware that implements the FMEC and $\mathrm{M}^{2} \mathrm{EC}$ at fixed edges and at mobile devices temporarily serving as local edges, respectively.

Our MEC middleware consists of two primary components:

i) the available Elijah platform (http://elijah.cs.cmu.edu/), one of the most promising and complete open-source project to realize a general-purpose support for MEC. Elijah supports a subset of basic OpenStack mechanisms for cloud resource management, including functions to migrate computing/storage resources in the form of VM near the dynamically identified edges of the network. In addition, basic mechanisms for VM synchronization and orchestration in the proximity of mobile device localities are included [14];

ii) our Elijah-based original extension module, called HEC middleware, that relevantly increases the basic MEC support with facilities for a) runtime enabling of $\mathrm{M}^{2} \mathrm{EC}$ features at selected mobile devices, b) (Docker-oriented) container support for more lightweight migration of computing/storage functions at HEC edges, and c) complete decoupling of regular mobile devices and their Elijah-based support at edges, e.g., via seamless and transparent management of needed $\mathrm{VM}$ /container migration in the case of mobile device handoffs between different edges during service provisioning [14].

In particular, concerning the dynamic migration of $\mathrm{VM} /$ containers (that typically realize MCS services) at HEC nodes, our middleware implements two types of related support, either proactive or reactive. On the one hand, the proactive migration allows to minimize and to automate in an efficient way virtualized function migration, by pre-loading the needed functions in advance on target HEC 
nodes. For instance, this approach is exploited to support end-user services with continuity requirements when device handoff prediction algorithms can be used with an adequate success probability. In this case, $\mathrm{VM}$ /container migration is started before receiving explicit location update by mobile devices due to their actual handoff procedures (note that $\mathrm{VM} /$ containers may be synthesized for migration purposes from scratch or by considering only the modified overlays/slices to enable more lightweight migrations [14]). On the other hand, our reactive migration support is triggered when a mobile device requests explicitly to move a virtualized function from one HEC edge to one another due to a device handoff (note that this is typically the solution we use when there is temporary unavailability of connectivity to the cloud, where typically sophisticated handoff prediction algorithms are run). Once triggered, our HEC middleware checks the possibility to execute the virtualized function required on the targeted MEC node and forwards to lower layer Elijah platform the request for the creation of a VM/container to execute the task. Finally, the HEC middleware returns to the mobile device the reference to transparently invoke the specified $\mathrm{VM} /$ container for future direct $\mathrm{VM} /$ container-to-device interactions.

By focusing on our HEC middleware (currently based on Android OS) at mobile devices, first of all we distinguish between regular mobile devices (capable of working only as service clients) and powerful ones (which may be promoted dynamically to host virtualized functions and to serve as $\mathrm{M}^{2} \mathrm{EC}$ nodes). In our current implementation, the latter are statically identified based on their device hw/sw profiles (at the moment high-end tablets or laptops that are locally paired with smartphones, typically of the same human owner, acting as MCS sensors). The evolution trend of mobile/embedded devices is such that the potential set of mobile nodes that can be promoted to $\mathrm{M}^{2} \mathrm{EC}$ at runtime is ever increasing; this is the case for experiments showing that even limited RaspberryPI nodes can adequately host OpenStack ++ middleware [14]; on these nodes we assume that our HEC middleware is already installed before starting service provisioning.

From the MCS application perspective, the tasks that have been already implemented and experimented for execution at HEC nodes are i) video analysis/processing for face recognition that can trigger online alarms and ii) analytics on all or fused monitoring indicators over geographical areas of highest interest and density (data fusion, history-based processing of temporal series, ...).

\subsection{FMEC and $M^{2} E C$ Selection and Human-enabled VM/Container Migration}

Our architecture considers both spatial and temporal dimensions for selecting FMECs and $\mathrm{M}^{2}$ ECs by analyzing the human mobility for an observation period. Concerning FMECs, we exploit the temporal dimension by considering places of our region remaining mostly active during the whole 24 hours: these are spatial locations not subject stable along the time. To this purpose, we use 
the DBSCAN algorithm which clusters together the positions of the users [15]. Points lying within the distance $\varepsilon$ are considered part of the same cluster, a new cluster is detected only if it contains at least $\gamma$ different points. DBSCAN results with $\mathrm{K}$ distinct clusters, we purge them by filtering out those clusters reporting a low number of different users, specifically those clusters composed by positions reported only by few users. After such filtering process, we only considered $k \leq \mathrm{K}$ clusters.

For what concerns the $\mathrm{M}^{2} \mathrm{EC}$, we consider the temporal dimension by selecting those places of our region becoming active only during specific time intervals. In fact, our goal is dynamically (re)configure our cloud architecture according to the natural rhythm of a city. To this purpose, we analyze the human mobility during some temporal frames characterizing the typical phases of a working day. For each of the frames, we cluster together the user positions with a process similarly to the one described for the FMEC. Also in this case, DBSCAN results with H clusters of which we keep the top $h \leq \mathrm{H}$.

The next step is moving data from FMEC to $\mathrm{M}^{2} \mathrm{EC}$ and vice-versa, to this purpose we consider the humans (i.e., couriers), and the mobile devices they carry in their pockets equipped with our HEC middleware, as the primary actors that can be involved into the loop. In particular, we assume mobile devices provide different kinds of network interfaces (short, medium and broadband) as well as storage capacity to enable store-and-forward of VM/containers between FMEC and $\mathrm{M}^{2} \mathrm{ECs}$, as well as to replicate them across users joining at the same time the same $\mathrm{M}^{2} \mathrm{EC}$ so to increase the stability defined as the capability to maintain the VM/container active in the $\mathrm{M}^{2} \mathrm{EC}$. For the selection of couriers, we keep track of user mobility and prefers those users that have a more repetitive and predictable behavior: the more a user commutes from a FMES to a $\mathrm{M}^{2} \mathrm{EC}$, the more he/she is a good courier candidate.

Finally, since not all the FMECs are connected to all $\mathrm{M}^{2} \mathrm{ECs}$ during the 24 hours, we consider the possibility of saving bandwidth in the cloud-to-FMEC direction and consequently storage resources at FMECs. With a closer view to details, HEC implements a load balancing function that exploits mobility traces and past connectivity history (between FMECs and $\mathrm{M}^{2} \mathrm{EC}$ ) to select which $\mathrm{VMs}$ /containers to move from the cloud to the FMECs by applying a locality principle that preloads at the FMEC only those ones that are more likely to be store-and-forwarded by a courier toward a $\mathrm{M}^{2} \mathrm{EC}$.

\section{Experimental Results}

Our experiments are based on the ParticipAct dataset [6] that implements a MCS platform. The ParticipAct dataset includes data collected from about 170 students in the Emilia Romagna region 
(Italy) by including their mobility traces. In particular, ParticipAct users are equipped with Android smartphones running an app that tracks their locations using the Google lo-cation APIs and a fixed sampling interval of 2.5 minutes. All the data collected are stored in the ParticipAct backend that provides some basic analytics features, such as filtering out location samples with low accuracy and interpolation of user locations between consecutive samples. The data collection covers about 2 years overall, and in this work we consider a period of 1 month, from March to April 2014, during the spring 2014 semester to ensure that most of the participants move in the city.

Of course, the dynamics of a city change according to the vacation periods of the year (i.e., working vs vacation days) and of the week (i.e., working days, often with slightly different behaviors on Mondays/Fridays, vs. weekends); often these dynamics are predictable to some extent and we could have adapted all initial HEC configurations according to that. However, to demonstrate the applicability of the proposed approach, in the following we consider a baseline worsening scenario where we statically decided (based on a pragmatic analysis of the mobility traces) to use as HEC configuration parameters (i.e., $k=6$ and $h=6$ ) average values calculated over the all the days in the considered period. We refer to a baseline scenario where HEC parameters are not finely tuned according to the vacation/weekly dynamics of the targeted system. This scenario also allows us to show the strengths of the adopted approach.

\subsection{Identifying FMECs and $\mathrm{M}^{2} \mathrm{ECs}$}

In this first set of experimental results, we analyze the mobility traces with the objective of spotting places in which deploy FMEC (denoted with capital letter $E$ ) as well as $\mathrm{M}^{2} \mathrm{EC}$ (denoted as capital letter $P$ ) according to dynamics of human mobility. Both the MECs (Fixed and Mobile) have been identified by using an observation period of three weeks. Specifically, we use the first three weeks of the dataset (March 1 to March 23, 2014) to learn how people in ParticipAct move and, therefore, identify places to consider for possible deployments of FMECs and $\mathrm{M}^{2}$ EC. Fig. 2 shows 6 FMECs selected as well as the centroid of each of the clusters marked as a red circle (E1 to E6). 


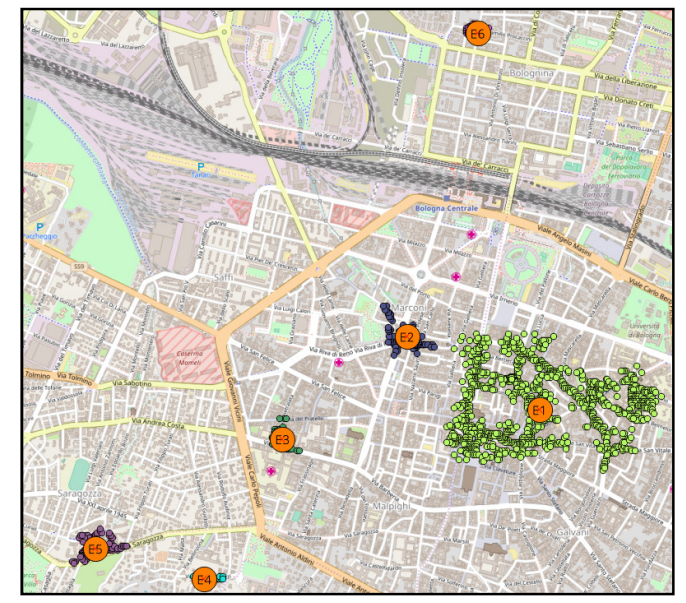

Figure 2. FMECs identified as DBSCAN clusters.

Concerning the $\mathrm{M}^{2} \mathrm{EC}$, we analyze those places of our sensing region becoming active only during specific time intervals. To this purpose, we analyze the mobility traces by slicing the dataset in 5 temporal frames: $9.00 \mathrm{AM}$ to $1 \mathrm{PM}, 1 \mathrm{PM}$ to $5 \mathrm{PM}, 5 \mathrm{PM}$ to $8 \mathrm{PM}, 8 \mathrm{PM}$ to $00 \mathrm{PM}$ and $00 \mathrm{PM}$ to 9.00AM. For each of such time intervals, we obtain 6 clusters reported on Fig. 3 (the $\mathrm{M}^{2} \mathrm{EC}$ centroids are not reported for better readability of the figure).
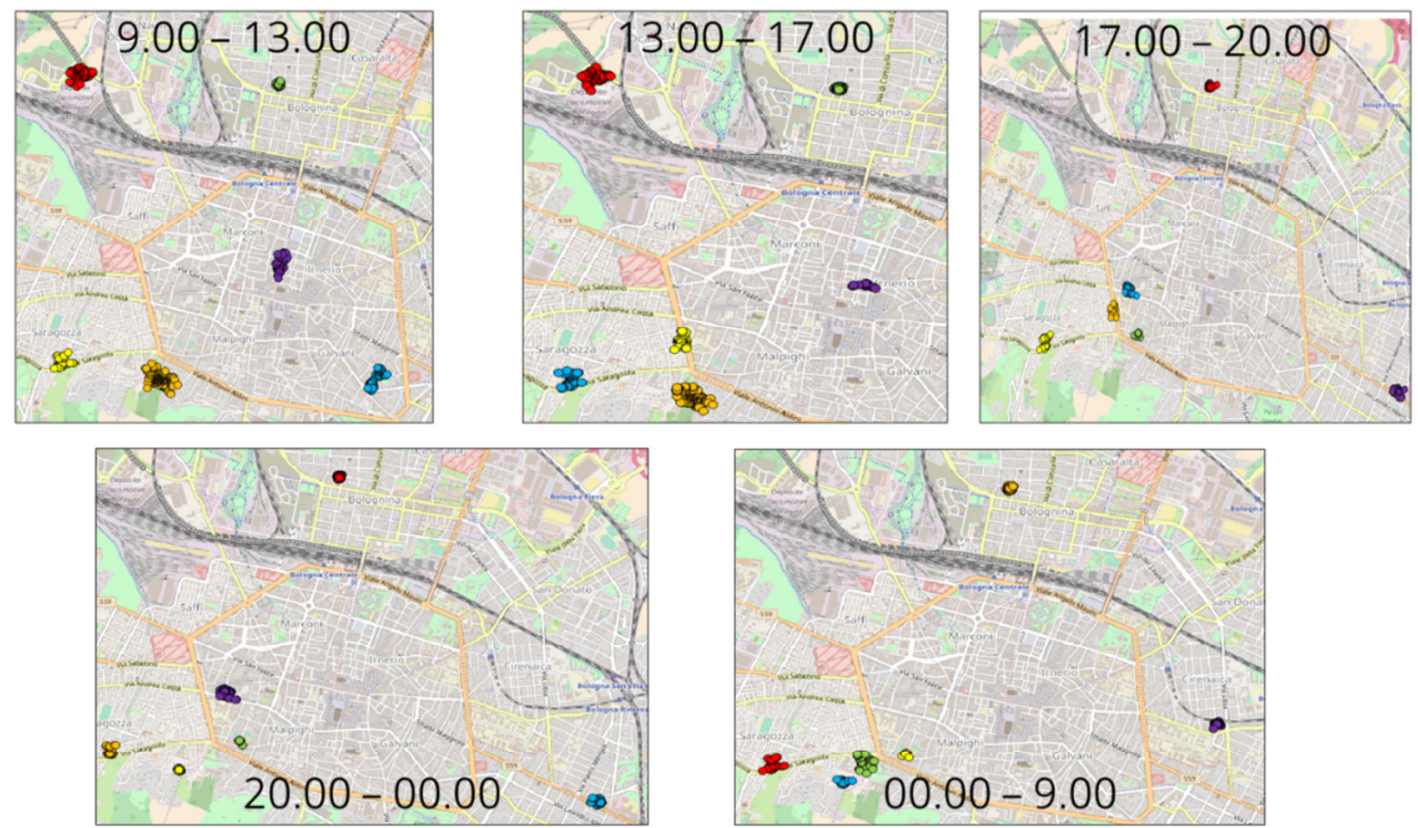

Figure 3. $\mathrm{M}^{2} E C$ identified as DBSCAN clusters.

\subsection{Assessing HEC Connectivity, Stability and Load Balancing}

This second set of experiments, differently from the experimental results presented in the previous subsection, is based the $4^{\text {th }}$ week of the dataset (March 24 to March 30, 2014) to validate the MEC selection previously done with respect to the mobility of $4^{\text {th }}$ week of our dataset. We aim 
at validating the effectiveness of our HEC implementation; we start showing some performance measures to quantify the connectivity and stability metrics as previously defined, and then assessing the performance improvements obtainable via FMEC load balancing.

Connectivity measures the robustness of links connecting FMEC and $\mathrm{M}^{2} \mathrm{EC}$. A link between two MECs exists if users move from/to a FMEC toward a $\mathrm{M}^{2} \mathrm{EC}$. The robust links are those characterized by a high number of users visiting FMEC and $\mathrm{M}^{2} \mathrm{EC}$ during the same time interval, while weak links are those MECs sporadically connected by few users. Connectivity is an interesting marker useful to understand, and to predict, the capability of the HEC architecture to remain connected along the time. Indeed, if during a specific time interval a pair of FMEC and $\mathrm{M}^{2} \mathrm{EC}$ remain disconnected, then the data stored on the FMEC cannot be moved to the $\mathrm{M}^{2} \mathrm{EC}$, giving rise to a sort of temporal disconnection of the cloud.

We measure the connectivity of the MECs previously identified, as a temporal graph, which is a graph evolving over the time. Fig. 4 shows, with a circular layout, FMECs (E1 to EO) are always the same, while $\mathrm{M}^{2} \mathrm{ECs}$ ( $P 1$ to $P 6$ ) may change for each of the 5 intervals as shown in Fig. 3 . The weight of the links connecting FMEC and $\mathrm{M}^{2} \mathrm{EC}$ (represented by different thickness of lines in Fig. 4) is proportional to the number of users whose position has been reported in both MECs during the same interval.
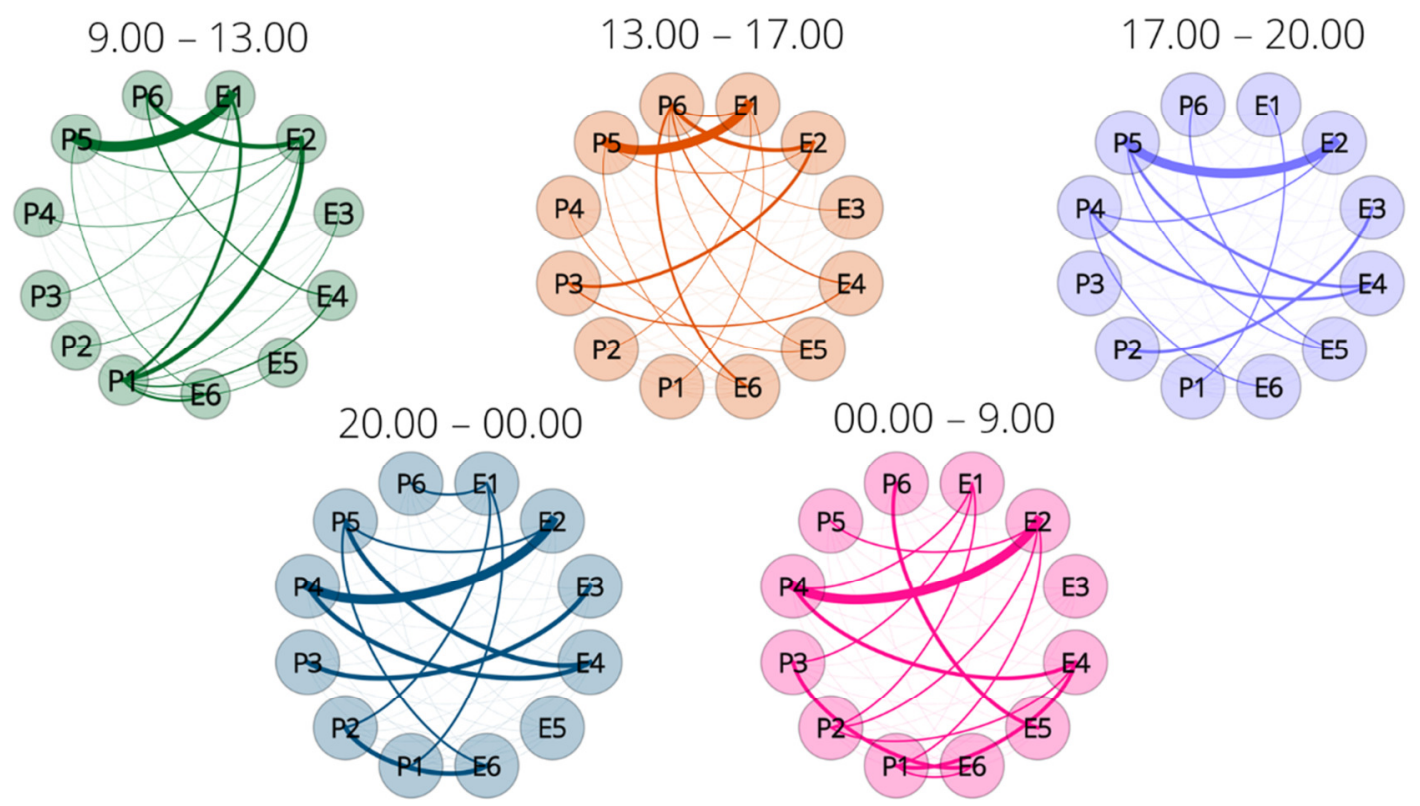

Figure 4. Measure of the connectivity as a temporal graph.

For example, E1 and P5 during 9.00 - 13.00 are well connected, while the link connecting E1 and $P 3$ during the same interval is weak. A first consideration is that it is possible to identify some 
dominant FMECs, namely those FMECs remaining well connected during the whole 24 hours. Examples of such FMECs are E1, E2, E4 and E6. These are FMEC deployed in core areas of the city representing a sort of backbone of our architecture. Secondly, we observe that during the 24 hours all the $\mathrm{M}^{2} \mathrm{EC}$ ( $P$ points on Fig. 4) are connected with at least one FMEC. Finally, we note that connectivity among MECs is significantly robust also during evening and night intervals (20.00 0.00 and $0.00-9.00$ ) ensuring the possibility of moving VMs/containers from/to FMECs also when user mobility tend to reduce.

The second metric we analyze refers to the stability of our cloud architecture, which measures the capability of $\mathrm{M}^{2} \mathrm{ECs}$ to maintain locally those data previously moved from a FMEC. In particular, we aim at measuring if, once data are moved from to a $\mathrm{M}^{2} \mathrm{EC}$, such data remain on the MEC for a while. To this purpose, we consider that users joining to a $\mathrm{M}^{2} \mathrm{EC}$ can exchange data directly by exploiting short, medium, high range network interfaces; specifically, we consider three kinds of direct mode communications: Bluetooth (up to 25 meters), Wi-Fi direct (up to 150 meters) and LTE direct (up to 500 meters). Given our 5 time intervals, we measure the percentage of time during which at least two users join to the same $\mathrm{M}^{2} \mathrm{EC}$. Fig. 5 shows, for each $\mathrm{M}^{2} \mathrm{EC}$, a triplet of histograms of different colors: orange represents stability via Bluetooth interface, green stability via Wi-Fi direct and blue the stability obtained via LTE direct interfaces; the black line on the left, instead, report the $100 \%$ scale.
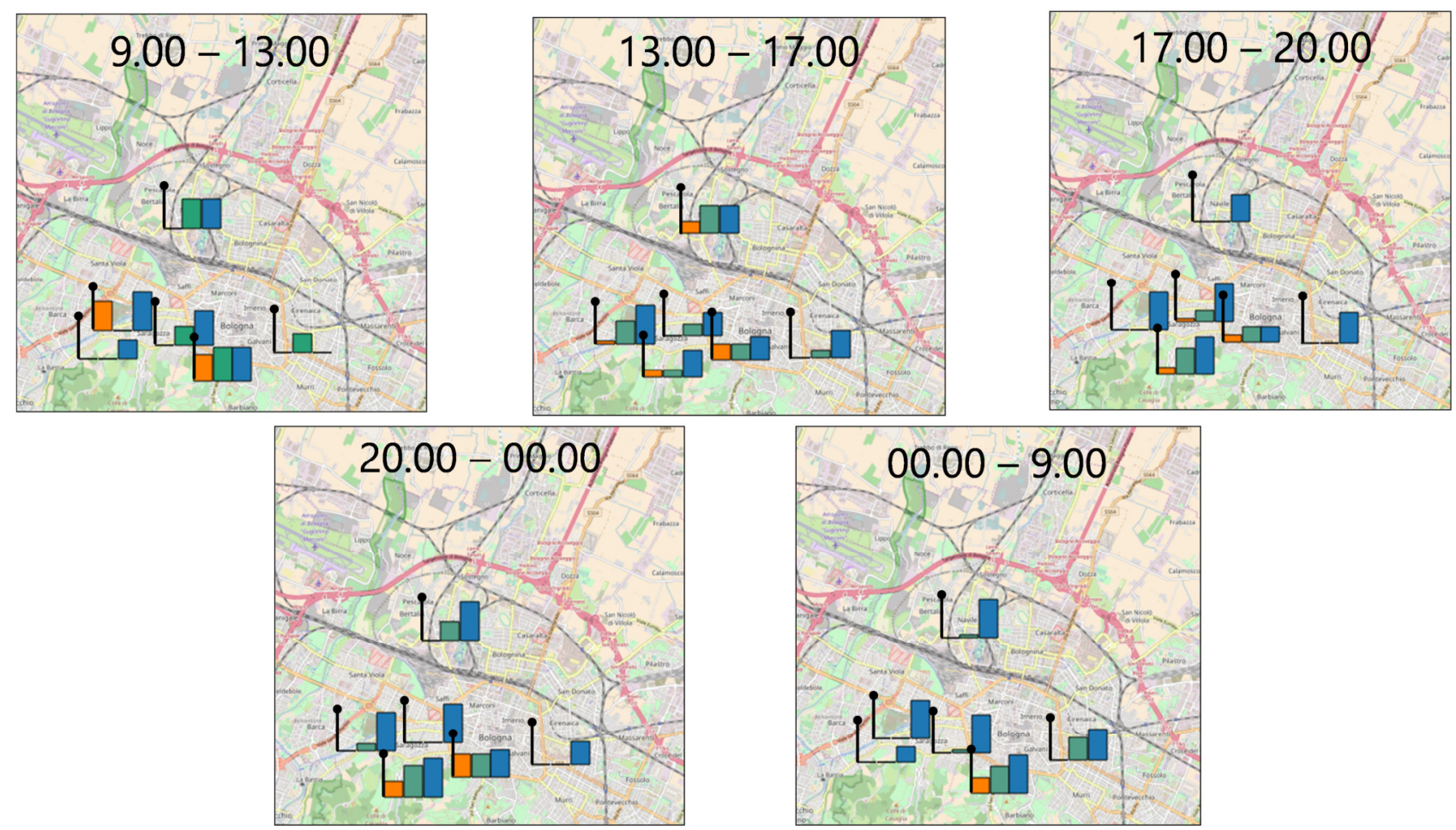

Figure 5. Stability of the $\mathrm{M}^{2} \mathrm{ECs}$ with different communication modes. 
We observe that stability is preserved for each of the $\mathrm{M}^{2} \mathrm{ECs}$ during the time intervals. There are cases in which the stability can be preserved only by means of high range interfaces, such as the $\mathrm{M}^{2} \mathrm{EC}$ deployed on the north region of Fig. 5 at interval 17.00 - 20.00: in this case, users use LTE direct connections in order to enable local replication. Differently, there are situations in which stability is guaranteed for all the network interfaces, such as the $\mathrm{M}^{2} \mathrm{EC}$ deployed on the south region of Fig. 5 during the interval $20.00-00.00$.

The last metric we present, refers to HEC load balancing across FMECs described in Section 3.2. In particular, we measure the benefits of balancing the load by focusing on the FMEC-to- $\mathrm{M}^{2} \mathrm{EC}$ direction and analyzing, for each of the 6 FMECs, how many M $^{2}$ ECs they are connected to via at least one courier. More specifically, given the FMEC $E_{i}(i=1$ to 6$)$ we measure the percentage $p$ of $\mathrm{M}^{2} \mathrm{ECs}$ for which there is no need to pre-cache $\mathrm{VMs} /$ containers since no connection from $E_{i}$ are available to such $\mathrm{M}^{2} \mathrm{ECs}$; this percentage ranges from $43 \%$ for $E_{2}$ up to $93 \%$ for $E_{5}$ and therefore we consider it a promising strategy to obtain significant performance improvements by avoiding unnecessary waste of bandwidth and storage resources at FMECs.

Let us conclude remarking that experimental results presented in this section are based on the analysis of the ParticipAct mobility dataset, but from our experience on mobility datasets (i.e., [7]) we believe that the same methodology can be adopted with other different following the same reproducible steps to cluster user positions along time, and then on spotting those locations activating during specific time widows or remaining active for long time.

\section{Conclusions}

In this paper, we presented HEC, a new model to ease the provisioning and to extend the coverage of traditional MEC solutions by bringing together the best of MEC and MCS research areas. The cornerstone of our proposal lies in the ability to leverage human sociality and mobility effects to broaden the MEC coverage through the impromptu formation of $\mathrm{M}^{2} \mathrm{ECs}$ and to make it more efficient and aware of the pulse of the city by dynamically tailoring the HEC hierarchical architecture to it. Moreover, results obtained from our ParticipAct living lab confirm us that clustering techniques can be employed to effectively plan both FMEC and $\mathrm{M}^{2} \mathrm{EC}$ placement.

Those encouraging results are pushing us to further investigate and refine our HEC model and we are currently exploring various main areas. On the one hand, we are working to enable the selfadaptable fine tuning of our HEC middleware to the different dynamics and variations of the city pulse. On the other hand, we are investigating how to increase the connectivity between FMEC and $\mathrm{M}^{2} \mathrm{EC}$ considering also some additional user profiling information for the courier selection. 


\section{References}

[1] S. Wang et al., "A Survey on Mobile Edge Networks: Convergence of Computing, Caching and Communications", IEEE Access, vol. PP, no. 99, pp.1-1 doi:10.1109/ACCESS.2017.2685434.

[2] S. K. Datta, R. P. Ferreira da Costa, C. Bonnet and J. Härri, "oneM2M architecture based IoT framework for mobile crowd sensing in smart cities", in Proceedings of 2016 European Conference on Networks and Communications (EuCNC), 2016, pp. 168-173.

[3] K. M. S. Huq, S. Mumtaz, J. Rodriguez, P. Marques, B. Okyere and V. Frascolla, "Enhanced C-RAN Using D2D Network", IEEE Communications Magazine, vol. 55, no. 3, pp. 100-107, March 2017.

[4] T. X. Tran, A. Hajisami, P. Pandey and D. Pompili, "Collaborative Mobile Edge Computing in 5G Networks: New Paradigms, Scenarios, and Challenges", IEEE Communications Magazine, vol. 55, no. 4, pp. 54-61, April 2017.

[5] Pappalardo, L.; Simini, F.; Rinzivillo, S.; Pedreschi, D.; Giannotti, F.; Barabási, A.L., "Returners and Explorers dichotomy in Human Mobility", Nature Communications, vol. 6, Article 8166, 2015.

[6] G. Cardone, A. Cirri, A. Corradi, L. Foschini, "The ParticipAct Mobile Crowd Sensing Living Lab: The Testbed for Smart Cities”, IEEE Commununications Magazine, vol. 52 , no. 10, pp. 78-85, October 2014.

[7] M. Girolami, S. Chessa, A. Caruso, "On Service Discovery in Mobile Social Networks: Survey and Perspectives", Computer Networks, vol. 88, pp. 51-71, 2015.

[8] K. Kaur, T. Dhand, N. Kumar, S. Zeadally, "Container-as-a-Service at the Edge: Trade-off between Energy Efficiency and Service Availability at Fog Nano Data Centers", IEEE Wireless Communications, vol. 24, no. 3, pp. 48-56, 2017.

[9] K. Ha et al., "Adaptive VM Handoff Across Cloudlets", Technical Report CMU-CS-15-113, CMU School of Computer Science, 2015.

[10] E. Saurez et al., "Incremental Deployment and Migration of Geo-Distributed Situation Awareness Applications in the Fog", in Proceedings of ACM Distributed and Event-based Systems Int. Conf., pp. 258-269, 2016.

[11] M. Felemban, S. Basalamah, A. Ghafoor, "A Distributed Cloud Architecture for Mobile Multimedia Services", 2013.

[12] A. Ravi, S.K. Peddoju, "Handoff Strategy for Improving Energy Efficiency and Cloud Service Availability for Mobile Devices”, Wireless Personal Communications, vol. 81, no. 1, pp. 101-132, 2015. 
[13] M.G.R. Alam, et al., "Multi-agent and Reinforcement Learning Based Code Offloading in Mobile Fog", in Proceedings of International Conference on Information Networking (ICOIN), 2016.

[14] P. Bellavista, M. Solimando, A. Zanni, “A Migration-enhanced Edge Computing Support for Mobile Services in Hostile Environments - Lessons Learnt from Platform Implementation and Deployment", to appear in Proceedings of International Wireless Communications and Mobile Computing Conference, pp. 1-6, 2017.

[15] M. Ester, H. peter Kriegel, J.S, X. Xu, “A density-based algorithm for discovering clusters in large spatial databases with noise", in Proceedings of International Conference on Knowledge Discovery, AAAI Press, pp. 226-231, 1996. 